\title{
Emission Line Properties of GPS/CSS Galaxies
}

\author{
Anton M. Koekemoer, Christopher P. O’Dea and Stefi A. Baum \\ Space Telescope Science Institute, 3700 San Martin Drive, \\ Baltimore, MD 21218, USA \\ koekemoer@stsci.edu \\ odea@stsci.edu \\ sbaum@stsci.edu
}

Received 2003 February 20, accepted 2003 March 19

\begin{abstract}
We present initial results from a program that we have begun, aimed at increasing the number of GPS and CSS sources for which good spectroscopic data are available for a variety of emission lines. We combine new observational data with previously published results to investigate whether there are any relationships between the excitation state of the gas and the radio properties of the sources, retaining distinctions between GPS and GSS galaxies and quasars. In scenarios where the youngest, most compact sources interact most strongly with the gas, it is perhaps to be expected that this can have an effect on the overall excitation state of the gas, which could therefore correlate with radio properties such as turnover frequency that plausibly trace the source size and age. However, we find a degree of scatter between the turnover frequency and gas excitation state that is several times larger than the characteristic degree of uncertainty in our line ratio measurements. This would suggest that either there is no underlying relationship between turnover frequency and excitation state, or alternatively that any potential relationship is completely diluted by variations in the intrinsic properties of the gas from one source to the next, which would thus be an important factor in determining the source properties.
\end{abstract}

Keywords: galaxies: active — galaxies: jets — galaxies: ISM — quasars: emission lines

\section{Introduction}

A key question in the formation and evolution of radio galaxies concerns the extent to which the environment determines the overall development of the radio source. Dense ambient gas in the host galaxy can dramatically affect the propagation of the radio jets outward from the active nucleus. Two classes of objects that hold a vital key to this issue are the compact steep spectrum (CSS) and gigahertz peaked spectrum (GPS) radio galaxies (e.g., Fanti et al. 1990; O'Dea, Baum, \& Stanghellini 1991; Spencer et al. 1991; Dallacasa et al. 1995; O'Dea 1998). Both types of objects are as powerful as large-scale 'classical double' radio galaxies but are up to several orders of magnitude more compact: CSS sources typically have size scales $\lesssim 10-20 \mathrm{kpc}$, while GPS sources are often $\ll 1 \mathrm{kpc}$. This makes them excellent probes of the physical processes that occur as the radio jets propagate through the ISM in their host galaxies.

The physical properties of the medium through which the jets propagate can be studied directly by means of the line emission produced by the ionised gas. The luminosity, kinematics, and excitation state of the gas provide a wealth of details about its physical characteristics and the degree to which the gas is affected by interactions with the radio jets. Specifically, comparing these observed gas properties in detail with models of auto-ionising shocks (Dopita \& Sutherland 1995, 1996; Koekemoer 1996; Koekemoer \& Bicknell 1998) yields direct information on the extent to which jets are interacting with the gas. Lines with a wide range of ionisation potentials, including [OІII] $\lambda$ 5007, [OІІI] $\lambda$ 4363, [ОII] $\lambda$ 3727, $\mathrm{H} \beta$,
$[\mathrm{NeIII}] \lambda 3868$, and $[\mathrm{Nev}] \lambda 3426$, are good diagnostics of the physical processes responsible for energising the gas, for example AGN photoionisation or shock fronts associated with jet interactions.

Scenarios in which the initial development of the jets is strongly influenced by the dense gas immediately surrounding the nucleus are expected on the basis of theoretical considerations (e.g., Bicknell, Dopita, \& O'Dea 1997), and also suggested by the observed alignment effect seen in CSS sources (de Vries et al. 1999). As the source ages, the jets encounter progressively less dense gas as they propagate out through the galaxy, thus decreasing the dominance of shocks relative to other mechanisms (e.g., nuclear photoionisation) in larger sources. Thus, one might expect relationships between the overall excitation state of the gas, and radio properties related to the source age and size.

In this paper we report our initial results from a project that we have begun, that is aimed at extending our previous work to a larger sample drawn from the full set of known CSS and GPS galaxies. We also present results from new spectroscopic data that we have obtained for sources in the sample where the existing data were insufficient.

\section{Sample Description}

Our goal in this project is to extend as much as possible the number of CSS and GPS sources for which high quality spectroscopy is available. There exists a variety of samples of both GPS and CSS sources in the literature that have been drawn from a range of different radio surveys and according to various selection criteria 
(e.g., Spencer et al. 1989; Fanti et al. 1990; O'Dea et al. 1991; Stanghellini et al. 1997, 1998; O’Dea 1998; Snellen et al. 1998a). Optical imaging and spectroscopy on these sources have been carried out by several teams including Stanghellini et al. (1993), Gelderman \& Whittle (1994), O'Dea et al. (1996), Morganti et al. (1997), de Vries et al. (1995, 1997, 1998, 1999, 2000), and Snellen et al. (1996, 1998b, 1999). We began by selecting all those sources in the literature for which optical identifications and spectroscopic redshifts were available. We next employed a redshift cutoff of $z<0.5$, which helps to remove cosmological evolutionary effects from the final sample but also ensures that we obtain measurements for emission lines in the rest-frame wavelength range of interest to us, which primarily includes the high-ionisation line [OIII] $\lambda 5007$ and the wide range of blueward emission lines.

We subsequently examined the available published spectroscopic and imaging data for this subset of sources to determine whether or not new spectroscopic observations were needed. Sources were included in our observing program if they had no published emissionline fluxes despite having a published redshift, or if the flux in [OIII] $\lambda 5007$ was sufficiently strong $\left(>10^{-15}\right.$ $\operatorname{erg~s}^{-1} \mathrm{~cm}^{-2}$ ) that other lines should be observable to our $3 \sigma$ limit of $\sim 10^{-16} \mathrm{erg} \mathrm{s}^{-1} \mathrm{~cm}^{-2}$, even if there were no published fluxes for them. These lines of interest included $[\mathrm{Nev}] \lambda 3426,[\mathrm{NeIII}] \lambda \lambda 3868,3967$, [OII] $\lambda$ 3727, [OIII] $\lambda$ 4363, HeII $\lambda$ 4681, $\mathrm{H} \gamma(\lambda 4340), \mathrm{H} \beta$ ( $\lambda$ 4861), as well as lines redward of [OIII] $\lambda 5007$ including [OI] $\lambda 6300, \mathrm{H} \alpha(\lambda 6583),[\mathrm{NII}] \lambda \lambda 6548,6583$, and [SII] $\lambda \lambda 6717,6731$ if these were accessible in the lowerredshift sources.

\section{Observations}

We observed a total of 31 sources, consisting of 14 CSS and 17 GPS sources, at the NOAO KPNO $4 \mathrm{~m}$ telescope on two observing runs during 2-4 July and 31 October-1 November, 2000. We used the R-C Spectrograph with the $2048^{2} \mathrm{~T} 2 \mathrm{~KB}$ detector, together with the BL250 grating in first order to obtain low-dispersion spectroscopy of each source, with a spectral resolution of $\sim 2.8 \AA$ /pixel. The relatively high sensitivity of this system in the blue makes it the ideal choice to obtain maximal $\mathrm{S} / \mathrm{N}$ in the wavelength range of interest, particularly the blue oxygen and neon lines, for objects with redshifts up to $z \sim 0.5$. To avoid problems with spectral order overlap resulting from the relatively low dispersion of the grating, we restricted ourselves to investigating wavelengths in the range $\sim 3400-6800 \AA$ for the lowerredshift sources, increasing to $\sim 3800-7600 \AA$ for the higher-redshift sources, using blue blocking filters where necessary.

For each source we obtained observations at a single position angle, using a relatively wide slit width of $2^{\prime \prime}$ in order to maximise the detected counts from each object. Since the majority of the sources are only marginally resolved from the ground, our program focuses only on the integrated emission-line fluxes for each object. The $2^{\prime \prime}$ slit was also still sufficiently narrow so as not to substantially degrade the spectral resolution of the data, thereby enabling us to separate lines that are close together in wavelength. Our exposure times ranged from 1 to 2 hours per object, generally divided into at least 3 subexposures in order to facilitate removal of cosmic rays. At the beginning and end of each night we observed spectrophotometric standards, along with sky flats, biases, and darks, and quartz lamp flats during the daytime. Since we are predominantly interested in relative spectrophotometry, i.e., line ratios, absolute photometric stability was not essential, although the weather during our runs was mostly clear and appeared photometrically stable to better than $\sim 10-15 \%$. The data were reduced in IRAF using standard long slit spectrophotometric reduction procedures.

\section{Results and Discussion}

We employed Gaussian-line fitting techniques to measure line fluxes in the spectrum of each object, and obtained chi-squared flux error estimates based on variance values propagated through the reduction process. We were able to measure line fluxes for both [OIII] $\lambda 5007$ and $\mathrm{H} \beta$ in 13 CSS and 11 GPS sources, and in addition were able to measure fluxes for [OII] $\lambda 3727$, [NeIII] $\lambda \lambda$ 3868,3967, and $[\mathrm{Nev}] \lambda 3426$ in about half of the sources. The full results from this work will be presented in a forthcoming paper (A.M. Koekemoer, C.P. O'Dea, \& S.A. Baum, in preparation); here we present the preliminary results as they currently stand.

Generally, the measured line ratios for the sources in the literature are also representative of emission integrated over the entire source, and are thus compatible with our measurements. Therefore, we combine our measurements with those from the literature in producing line ratio plots, as well as examining whether there are any relationships between the line ratios and the radio properties of the sources. Although the sources are drawn from heterogeneous samples, generally these samples are defined on the basis of radio properties alone, thus there are unlikely to be any explicit selection effects that may depend on emission-line properties. In our plots we differentiate between GPS and CSS sources, and also between objects that show quasar-like spectra and those that do not ('galaxies'). For quasars, our aim is consider only the narrow-line Balmer emission in our results, in order to afford a direct comparison with the emission-line properties of the galaxies. A few of the quasars have extremely strong broad-line emission, and it is possible that some of this may contaminate the narrow-line measurements despite our efforts to remove it; we discuss this in more detail below.

In Figure 1 we show the diagnostic plot of [NeIII] $\lambda \lambda 3868,3967$ against [OIII] $\lambda 5007 / \mathrm{H} \beta$ for our sources. This plot serves predominantly as a consistency check, since the ionisation potentials of [OIII] $\lambda 5007$ and [NeIII] $\lambda \lambda$ 3868,3967 are reasonably similar. The good agreement suggests that our line ratio measurements are reliable, since the two quantities are independently measured. We 


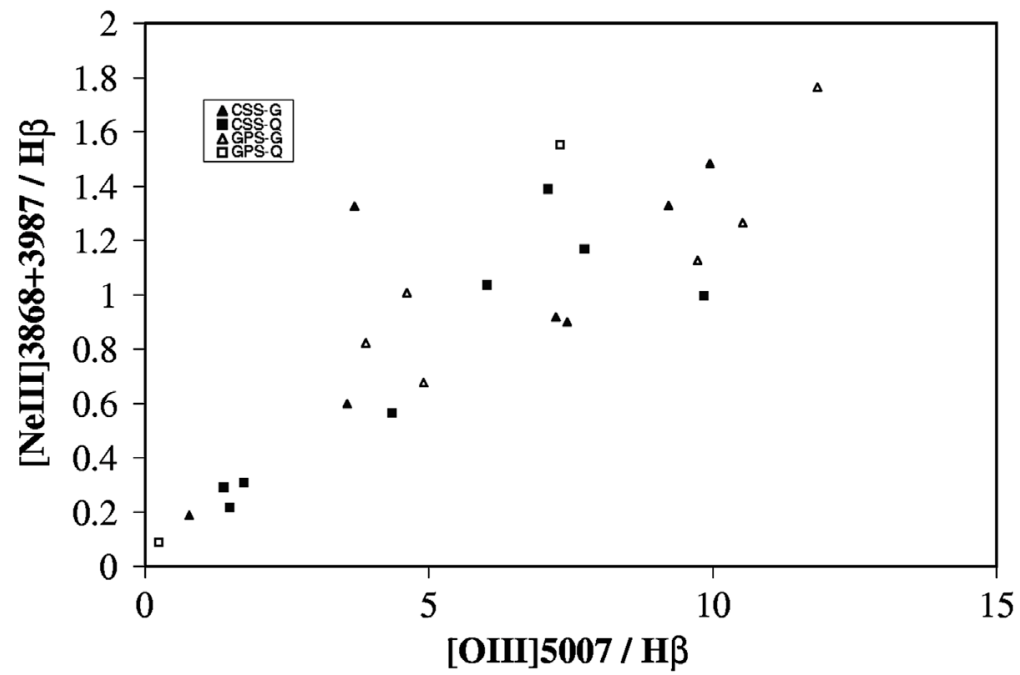

Figure 1 Plot of [NeIII] $\lambda \lambda$ 3868,3967 against [OIII] $\lambda$ 5007/H $\beta$ for our sample of CSS and GPS galaxies and quasars. This plot serves predominantly as a consistency check, showing that our measured line ratios agree with expected behaviour (high values of [OIII] should generally correspond with high values of $[\mathrm{NeIII}])$.

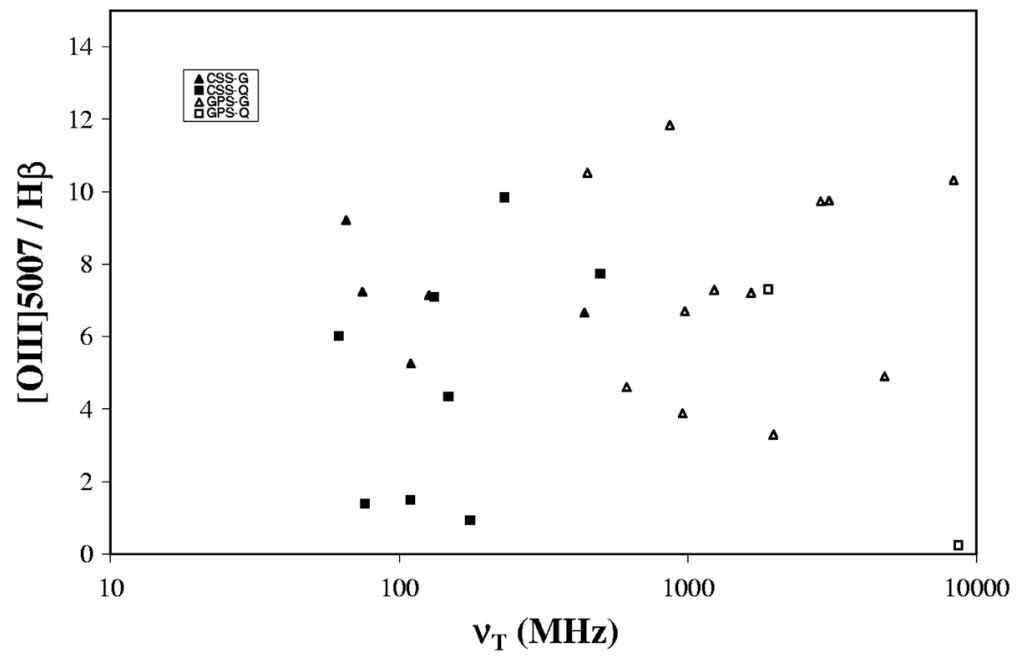

Figure 2 Plot of [OIII] $\lambda 5007 / \mathrm{H} \beta$ as a function of intrinsic turnover frequency $\nu_{\mathrm{T}}$, for our sample of CSS and GPS galaxies and quasars. There is no evidence of any strong trends between excitation state and turnover frequency; the scatter is several times larger than the characteristic uncertainty in our line ratio measurements as shown in Figure 1, thus any strong trends would have been detectable.

also note that for quasars, there is always the possibility of contamination of the narrow Balmer lines by emission from the broad-line region, which would tend to lower any ratios that use this line as a denominator. Indeed, four of the quasars do exhibit unusually low values ([OIII] $\lambda 5007 / \mathrm{H} \beta \lesssim 2$ ), suggesting some residual contamination from the broad-line region which happens to be unusually strong in these particular quasars. We expect that this will be remedied when we re-measure the lines using an approach of simultaneously fitting all the narrow lines in the spectrum (A.M. Koekemoer et al., in preparation). For the moment we simply note that these four quasars likely contain substantial broad-line $\mathrm{H} \beta$ contamination. There is a fifth object in this region, $0116+319$, classified as a CSS galaxy by Spencer et al. (1989); in our spectrum it exhibits no dominant broad-line emission but it does have unusually strong narrow Balmer lines. The remainder of the quasars in both the CSS and GPS subsamples fall well within the range of ionisation values occupied by the galaxies ([OIII] $\lambda 5007 / \mathrm{H} \beta \gtrsim 4$ ) which suggests for these objects we have successfully minimised the contribution from the broad-line emission and are measuring predominantly the extended narrow-line gas in these objects.

In Figure 2 we plot the excitation state of the gas, parameterised by [OIII] $\lambda 5007 / \mathrm{H} \beta$, against the intrinsic rest-frame turnover frequency $v_{\mathrm{T}}$ for each source. Sources with higher turnover frequency are generally observed to be more compact (e.g., O'Dea 1998 and references therein); we use this as a tracer of the source age in the 
context of the evolutionary scenario of Bicknell et al. (1997) where the youngest sources are the most compact. If the gas excitation properties were dominated by the evolutionary state of the radio source, for example with the youngest sources exhibiting the strongest shocks, then some correlation might be expected between the excitation state and the radio properties. However, no clear trend is visible in this plot for either the CSS or GPS galaxies or quasars - apart from the four quasars with low [OIII] $\lambda 5007 / \mathrm{H} \beta$ that we described earlier, the remainder of the sources are more or less uniformly distributed between [OIII] $\lambda 5007 / \mathrm{H} \beta \sim 4-12$, and do not display any strong trend with turnover frequency.

The lack of any strong correlation in Figure 2 is unlikely to be due to scatter from errors in our line ratio measurements, since we verified in Figure 1 the existence of a relatively tight relationship between two independently measured diagnostic lines that have similar ionisation potential. It is also unlikely to represent errors in the turnover frequency measurements from the literature, since these are generally very well constrained by observations at frequencies both above and below the turnover frequency (except possibly for the very highest $\nu_{\mathrm{T}}$ sources, where the turnover frequency is more appropriately regarded as a lower limit). Thus, we are led to the conclusion that either there is no intrinsic relationship at all between the excitation state and the radio jet properties, or alternatively that any possible relationship is completely diluted by intrinsic variations from one source to the next. For example, the intrinsic properties of the gas, including its density, neutral fraction, volume filling factor and spatial distribution, are also all important factors in determining its overall observed ionisation state. If these quantities change sufficiently from one source to the next, then the overall ionisation state will be determined not so much by the evolutionary state of the source, but rather by the intrinsic properties of the gas specific to each source.

\section{Summary}

We have reported on our initial results from a program of spectroscopic observations of all the known GPS and CSS sources, aimed at increasing the number of sources for which high $\mathrm{S} / \mathrm{N}$ measurements are available for a variety of emission lines corresponding to a range of excitation states. We combine new observational data with previously published results to investigate possible trends between the excitation state of the gas and the radio properties of the sources, retaining distinctions between GPS and GSS galaxies and quasars. On the basis of evolutionary models of GPS/CSS sources, one might expect that the youngest, most compact sources would exhibit the strongest shock interactions with surrounding gas, thereby influencing the observed excitation state. However, we find no strong trends between excitation state and radio parameters such as the spectral turnover frequency, with the plot exhibiting scatter on scales substantially larger than our degree of measurement uncertainty. This may suggest that either these parameters are not related at all, or even if they were, that any possible relationship would be completely diluted by variations in the intrinsic properties of the gas properties from one source to the next. Subsequent directions for this work will include measurements of a wider range of diagnostic lines, as well as other parameters related to the radio properties of the sources.

\section{References}

Bicknell, G. V., Dopita, M. A., \& O’Dea, C. P. 1997, ApJ, 485, 112 Dallacasa, D., Fanti, C., Schilizzi, R. T., \& Spencer, R. E. 1995, A\&A, 295, 27

de Vries, W. H. 1999, PhD Thesis, Groningen

de Vries, W. H., Barthel, P. D., \& Hes, R. 1995, A\&AS, 114, 259

de Vries, W. H., Barthel, P. D., \& O’Dea, C. P. 1997, A\&A, 321, 105

de Vries, W. H., O’Dea, C. P., Barthel, P. D., \& Thompson, D. J. 2000, A\&AS, 143, 181

de Vries, W. H., O’Dea, C. P., Baum, S. A., \& Barthel, P. D. 1999, ApJ, 526, 27

Dopita, M. A., \& Sutherland, R. S. 1995, ApJ, 455, 468

Dopita, M. A., \& Sutherland, R. S. 1996, ApJS, 102, 161

Fanti, R., Fanti, C., Schilizzi, R. T., Spencer, R. E., Rendong, N., Parma, P., van Breugel, W. J. M., \& Venturi, T. 1990, A\&A, 231,333

Gelderman, R., \& Whittle, M. 1994, ApJS, 91, 491

Koekemoer, A. M. 1996, PhD Thesis, Australian National University

Koekemoer, A. M., \& Bicknell, G. V. 1998, ApJ, 497, 662

Koekemoer, A. M., O’Dea, C. P. \& Baum, S. A., 2003, in preparation

Morganti, R., Tadhunter, C., Dickson, R., \& Shaw, M. 1997, A\&A, 326,130

O’Dea, C. P. 1998, PASP, 110, 493

O'Dea, C. P., Baum, S.A., \& Stanghellini, C. 1991, ApJ, 380, 66

O'Dea, C. P., Stanghellini, C., Baum, S. A., \& Charlot, S. 1996, ApJ, 470,806

Snellen, I. A. G., Bremer, M. N., Schilizzi, R. T., Miley, G. K., \& van Ojik, R. 1996, MNRAS, 279, 1294

Snellen, I. A. G., Schilizzi, R. T., de Bruyn, A. G., Miley, G. K., Rengelink, R. B., Röttgering, H. J., \& Bremer, M. N. 1998a, A\&AS, 131, 435

Snellen, I. A. G., Schilizzi, R. T., Bremer, M. N., de Bruyn, A. G., Miley, G. K., Röttgering, H. J., McMahon, R. G., \& Pérez Fournon, I. 1998b, MNRAS, 301, 985

Snellen, I. A. G., Schilizzi, R. T., Bremer, M. N., Miley, G. K., de Bruyn, A. G., \& Röttgering, H. J. 1999, MNRAS, 307, 149

Spencer, R. E., McDowell, J., Charlesworth, M., Fanti, R., Parma, P., \& Peacock, J. A. 1989, MNRAS, 240, 657

Spencer, et al. 1991, MNRAS, 250, 225

Stanghellini, C., O’Dea, C. P., Baum, S. A., \& Laurikainen, E. 1993, ApJS, 88, 1

Stanghellini, C., O’Dea, C. P., Baum, S. A., Dallacasa, D., Fanti, R., \& Fanti, C. 1997, A\&A, 325, 943

Stanghellini, C., O’Dea, C. P., Dallacasa, D., Baum, S. A., Fanti, R., \& Fanti, C. 1998, A\&AS, 131, 303 